\title{
PLASMON METAL NANOSTRUCTURES FORMATION IN PIEZOCOMPOSITE MATERIAL CONTROLLABLE IN MICROMETRIC LEVEL FOR DETECTION AND SENSING CELL-BIOLOGICAL PARTICLES
}

\author{
Tomas Janusas (PHD student), Arvydas Palevicius, Giedrius Janusas \\ Kaunas university of technology, Lithuania
}

The molecular interactions and cell-biological mechanisms behind the adhesive behaviour of a cell are not properly understood till now. In the last decades, the development of nanocomposite materials and the hope for elaboration of successful bacteria/virus or other particle detection accelerated the search for appropriate materials and conditions which may help to keep particles functioning and detect them on artificial surfaces [1].

One of solutions to detect those particles in biologically active medium is insertion of metal nanoparticles in polymer matrix. Designed metal/polymer based nanocomposites with microperiodic structures are like small sensing chips based on plasmonic effect and works like a lacmus paper - nanocomposite surface with metal nanoparticles is covered with investigated biologically active medium, antigen and antibody interaction appears. Bacteria/virus or other particles existing in observed medium are automatically attracted by metal nanoparticles to the surface of nanocomposite.

Nanocomposite materials having piezoelectric properties are a field of investigations, because of their scientific significance and technological implications. From both scientific and technological points of view, it is highly desirable to pattern piezoelectric nanocomposite films with feature size down to micro- or nanometer scale.

The microperiodic structure on nanocomposite surface couples the light of a $\mathrm{He}-\mathrm{Ne}$ laser at a given resonance angle into the waveguide layer. This incoupling angle is very sensitive to the presence of adsorbed particles and to any change of refractive index of the medium covering the surface of the nanocomposite. By precise measurement of the incoupling angle, the adsorbed particles can be determined with ultrahigh sensitivity.

When sensing chip (microperiodic structure on nanocomposite layer) is in contact with biologically active medium (liquid (i.e. blood) or gas (i.e. $\left.\mathrm{CO}_{2}\right)$ ), it is essential that particles in that medium would be attracted to the surface of nanocomposite in order to detect them.

But because of too high concentration of investigated medium or too big size of particles in that medium, metal nanoparticles are not able automatically to attract them to nanocomposite surface. 
Because of the properties of piezocomposite material controllable in micrometric level by ultrahigh frequency excitation the mathematical model of the system is developed. This model allows to analyse the mechanical parameters of the system which enable to control motion of cell-biological mechanisms in bio suspension and to force them to be attracted by designed microperiodic structure on nanocomposite layer in order to be prepared for analysing them using single molecule analytical tools.

The optical, electrical and mechanical characteristics of created piezocomposite material controllable in micrometric level and the design and functionality of the microperiodic structure on nanocomposite layer to be used for single molecule analysis methodology are presented.

\section{References}

1. Single-molecule biosensors: Recent advances and applications. Namik Akkilic, Stefan Geschwindner Biosensors and Bioelectronics (2020).

Acknowledgement. This research was funded by a Grant S-MIP-19-49 from the Research Council of Lithuania. 